\title{
OPEN Effect of pressure controlled volume guaranteed ventilation during pulmonary resection in children
}

\author{
Change Zhu ${ }^{1,3}$, Rufang Zhang ${ }^{2,3}$, Shenghua Yu ${ }^{1}$, Yuting Zhang ${ }^{1}$ \& Rong Wei ${ }^{1 \bowtie}$
}

The purpose of the study was to evaluate the effect of pressure controlled volume guaranteed ventilation in children requiring one lung ventilation during pulmonary resection. Patients were randomly assigned to the lung protective ventilation combined with pressure controlled volume guaranteed group (PCV-VG group) or the lung protective ventilation combined with volume controlled ventilation group (VCV group). Both groups received tidal-volume ventilation of $8 \mathrm{ml} \mathrm{kg}^{-1}$ body weight during two lung ventilation and $6 \mathrm{ml} \mathrm{kg}^{-1}$ during $\mathrm{OLV}$, with sustained $5 \mathrm{cmH}_{2} \mathrm{O}$ positive end-expiratory pressure. Data collections were mainly performed at $10 \mathrm{~min}$ after induction of anaesthesia during TLV (T1), 5 min after OLV initiation (T2) and 5 min after complete CO2 insufflations (T3). In total, 63 patients were randomly assigned to the VCV $(n=31)$ and PCV-VG $(n=32)$ groups. The PCV-VG group exhibited lower PIP than the VCV group at T1 $\left(16.8 \pm 2.3\right.$ vs. $\left.18.7 \pm 2.7 \mathrm{cmH}_{2} \mathrm{O}, P=0.001\right), \mathrm{T} 2(20.2 \pm 2.7$ vs. $\left.22.4 \pm 3.3 \mathrm{cmH}_{2} \mathrm{O}, P=0.001\right)$, and T3 $\left(23.8 \pm 3.2\right.$ vs. $\left.26.36 \pm 3.7 \mathrm{cmH}_{2} \mathrm{O}, P=0.01\right)$. Static compliance was higher in the PCV-VG group at T1, T2, and T3 $(P=0.01)$. After anaesthesia induction, lung aeration deteriorated, but with no immediate postoperative difference in both groups. Postoperative lung aeration improved and returned to normal from $2.5 \mathrm{~h}$ postextubation in both groups. $\mathrm{PH}$ was lower and $\mathrm{PaCO}_{2}$ was higher in VCV group than PCV-VG group during one lung ventilation. No differences were observed in $\mathrm{PaO}_{2}-\mathrm{FiO}_{2}$-ratio at $\mathrm{T} 2$ and $\mathrm{T} 3$, the incidence of postoperative pulmonary complications, intraoperative desaturation and the length of hospital stay. In paediatric patients, who underwent pulmonary resection requiring one lung ventilation, PCV-VG was superior to VCV in its ability to provide lower PIP, higher static compliance and lower $\mathrm{PaCO}_{2}$ at one lung ventilation during pneumothorax. However, its beneficial effects on different pathological situations in pediatric patients need more investigation.

One lung ventilation (OLV) has been widely used in children; however, it is associated with increased postoperative pulmonary complications according to adult research ${ }^{1}$. OLV is recognized as a risk factor for acute lung injury $(\mathrm{ALI})^{2}$. ALI and acute respiratory distress syndrome are the leading causes of death after thoracic surgery, and they significantly reduce 1 -year survival rate ${ }^{3}$. Paediatric patients have smaller functional residual capacities and larger closing volumes, rendering them having high airway pressure, low lung compliance, especially during one lung ventilation ${ }^{4}$. Children are vulnerable to ventilator induced barotrauma owing to high airway pressures during $\mathrm{OLV}^{5}$, which brings great concern to pediatric anesthesiologist ${ }^{4}$. Pressure controlled volume guaranteed ventilation (PCV-VG) is a novel type of ventilation mode. The decreasing airflow of PCV-VG allows airway pressure to achieve its maximum value at the beginning of inhalation and sustains the entire inhalation phase. Continuous plateau pressure in PCV-VG is more conducive for oxygen diffusion ${ }^{6}$. To date, several studies involving adults have demonstrated that PCV-VG potentially reduces airway pressure and improves lung compliance compared to volume controlled ventilation $(\mathrm{VCV})^{6-8}$; however, the anatomical and physiological characteristics of children differ from adults. Whether children benefit from PCV-VG is unclear and relevant studies are lacking. On this premise, this study aimed to compare PCV-VG with VCV in terms of airway pressure, static compliance, $\mathrm{PaO}_{2}-\mathrm{FiO}_{2}$-ratio, $\mathrm{PaCO}_{2}$, arterial $\mathrm{pH}$, lung aeration in lung ultrasound, postoperative pulmonary complications, intraoperative desaturation, hospital stay, and haemodynamics in children requiring OLV.

${ }^{1}$ Department of Anesthesiology, Shanghai Children's Hospital, Shanghai, China. ${ }^{2}$ Department of Cardiothoracic Surgery, Shanghai Children's Hospital, Shanghai, China. ${ }^{3}$ These authors contributed equally: Change Zhu and Rufang Zhang. ${ }^{\varpi}$ email: Rongwei2014@163.com 


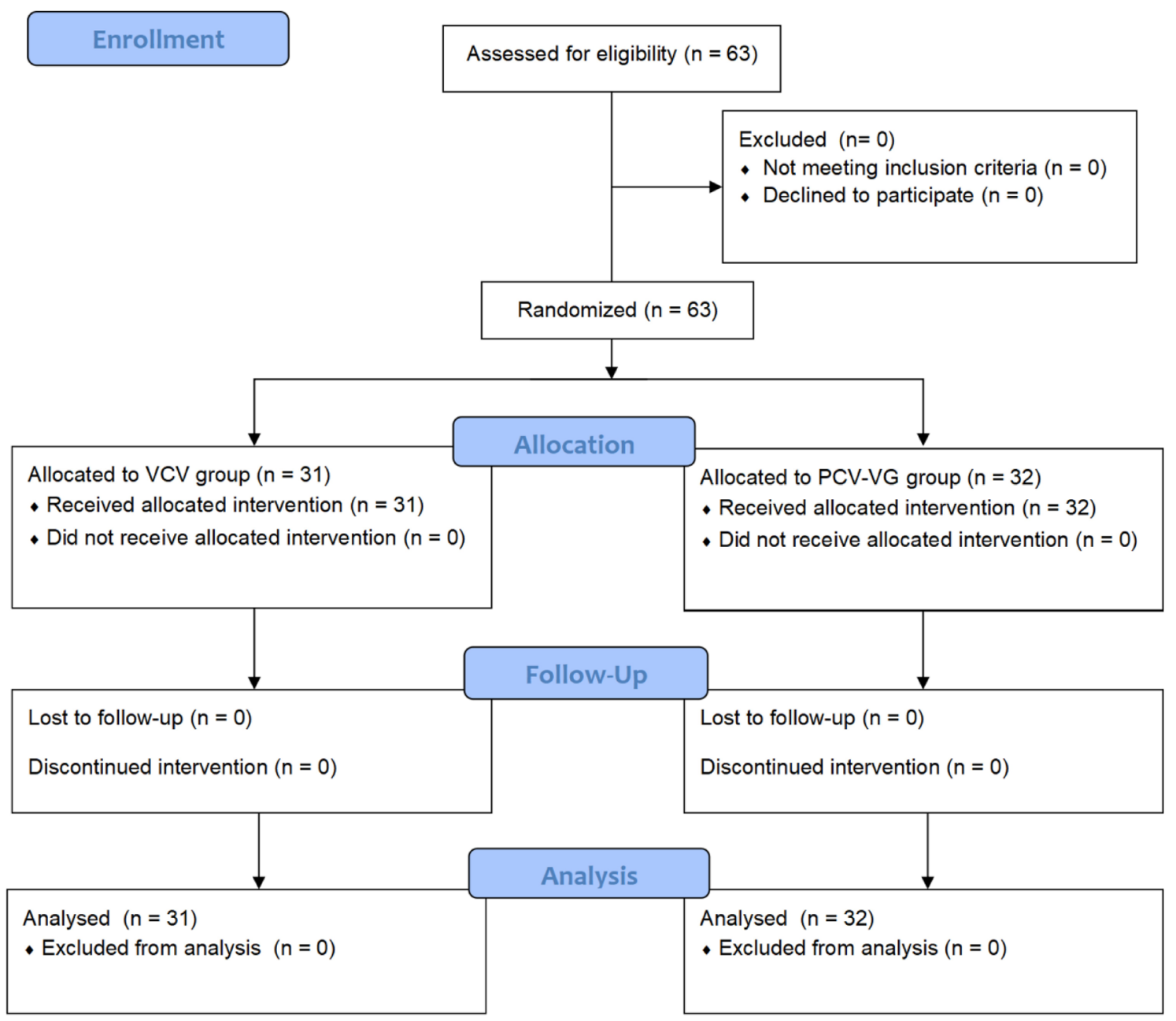

Figure 1. CONSORT flow diagram for patients included in the study.

\section{Methods}

The study was conducted in accordance with the principles of the Declaration of Helsinki ${ }^{9}$ after receiving approval from the Ethics Committee of Shanghai Children's Hospital, Shanghai, China on 24 July 2019 (approval number: 2019R044-F01). The trial was registered in the Chinese Clinical Trial Registry at www.chictr.org.cn (trial number: ChiCTR2000035189, 02/08/2020). Informed consent was obtained from the parents or legal guardians of the children. This single-centre, prospective, randomised, controlled trial was conducted at a tertiary teaching children's hospital affiliated with Shanghai Jiao Tong University, China, from 7 August 2020. The enrolment and allocation of patients are summarised in a CONSORT flow diagram (Fig. 1).

Inclusion criteria were as follows: Healthy paediatric patients aged 0-5 years with American Society of Anesthesiologists physical status 1 or 2 who required OLV while undergoing thoracoscopic surgery because of congenital cystic adenomatoid malformation. The exclusion criteria severe heart disease, other lung disease, upper respiratory tract infections, difficult airway or tracheotomy.

Computer-generated, sealed-envelope randomisation was performed to assign patients to one of the following two parallel arms in a 1:1 ratio, receiving different mechanical ventilation protocols: lung protective ventilation (LPV) combined with PCV-VG (PCV-VG group) or LPV combined with VCV (VCV group). One investigator (SZ) opened the envelopes and performed different mechanical ventilation protocols. The investigator did not participate in any other aspects of the trial. The patients as well as the Data Safety and Monitoring Board (DSMB) were also blinded to the random allocation.

All patients received a standardised general anaesthetic protocol, which included pre-oxygenation (without continuous positive airway pressure) and intravenous fentanyl $\left(2 \mu \mathrm{g} \mathrm{kg}^{-1}\right)$, propofol $\left(3 \mathrm{mg} \mathrm{kg}^{-1}\right)$, and rocuronium $\left(0.6 \mathrm{mg} \mathrm{kg}^{-1}\right)$. 5-Fr bronchial blocker (BB) was placed outside the endotracheal tube and placed into the target bronchi using fibre-optic bronchoscope. The pressure of the tracheal intubation cuff was maintained at 20-30 $\mathrm{cmH}_{2} \mathrm{O}^{10}$. All patients received ventilation using the same type of mechanical ventilator (Datex-Ohmeda-Avance CS2 Anesthesia Machine, GE Healthcare, Madison WI USA). At the end of one lung ventilation, the lungs were re-expanded manually with sustained inflation with $20-30 \mathrm{cmH}_{2} \mathrm{O}$ of positive airway pressure for $10-15 \mathrm{~s}$ under direct observation to restore two lung ventilation ${ }^{4,11}$. 
Anaesthesia was maintained using propofol $5-8 \mathrm{mg} \mathrm{kg}^{-1} \mathrm{~h}^{-1}$ to maintain the BIS (Philips Healthcare, Andover, MA) at 40-60 and remifentanil $0.1-0.4 \mu \mathrm{g} \mathrm{kg}^{-1} \mathrm{~min}^{-1}$ to maintain haemodynamic stability. Crystalloid solutions $\left(6-8 \mathrm{ml} \mathrm{kg}^{-1} \mathrm{~h}^{-1}\right)$ were used as maintenance fluids intraoperatively.

Ventilation settings in both groups were as follows:

Mechanical ventilation started with a tidal volume of $8 \mathrm{ml} \mathrm{kg}{ }^{-1}$ with PEEP at $5 \mathrm{cmH}_{2} \mathrm{O}$ in both groups during two lung ventilation (TLV) and $6 \mathrm{ml} \mathrm{kg}{ }^{-1}$ with PEEP at $5 \mathrm{cmH}_{2} \mathrm{O}$ in both groups during one lung ventilation. Volume controlled mode was used for mechanical ventilation in the VCV group and pressure controlled volume guaranteed ventilation mode was used in the PCV-VG group. The tidal volume was set based on the actual body weight. The inspired oxygen fraction was 0.5 during TLV and 1 during OLV. To ensure the end-expiratory concentration at $4.7-5.3 \mathrm{kPa}$, respiratory rate was adjusted at 18-25 breaths/min during TLV with an inspiratory/ expiratory (I/E) ratio of 1:2. Respiratory rate was adjusted at $25-30$ breaths $\mathrm{min}^{-1}$ during OLV with an $\mathrm{I} / \mathrm{E}$ ratio of 1:2 to maintain an ETCO2 of no more than $7.9 \mathrm{kPa}$.

Measurements:

Data collections were performed during the following time points:

- Before induction of anaesthesia (T0).

- 10 min after induction of anaesthesia during TLV (T1).

- 5 min after OLV initiation (T2).

- 5 min after complete $\mathrm{CO}_{2}$ insufflations (T3).

- After wound closure before extubation (T4).

- $2.5 \mathrm{~h}$ after surgery (T5).

The following data were collected or calculated:

- Peak inspiratory pressure (PIP), static compliance.

- $\mathrm{PaO}_{2}-\mathrm{FiO}_{2}$-ratio, $\mathrm{PaCO}_{2}$ and arterial $\mathrm{pH}$.

- Intraoperative desaturation (peripheral oxygen saturation $\left(\mathrm{SpO}_{2}\right)<90 \%$ ).

- Postoperative pulmonary complications.

According to the recommendation of European joint taskforce published guidelines for perioperative clinical outcome (EPCO), the pulmonary complications were defined as respiratory infection, respiratory failure, pleural effusion, atelectasis, pneumothorax, bronchospasm, and aspiration pneumonitis ${ }^{12,13}$.

- Lung ultrasonography (LUS) in the dependent lung.

As reported in our previous study, LUS is an accurate method for diagnosing anesthesia-induced atelectasis in children. For the diagnosis of atelectasis, the sensitivity and accuracy of LUS was $88 \%$ and the specificity was $89 \%$ compared with MRI ${ }^{14,15}$. LUS was performed at the following three specific intervals: immediately before induction of anaesthesia (T0), immediately after wound closure before extubation (T4), $2.5 \mathrm{~h}$ after surgery (T5).

The four levels of aeration in LUS examination were classified as follows: $\mathrm{N}=0, \mathrm{~B} 1=1, \mathrm{~B} 2=2$, and $\mathrm{C}=3^{4,16,17}$.

Statistical analysis. Data are expressed as $\mathrm{n}(\%)$, mean $\pm \mathrm{SD}$, or median (IQR) depending on the distribution of the data. Comparison of continuous variables between the study groups was performed using Student's $\mathrm{t}$-test for normally distributed data or the Mann-Whitney $U$-test for non-normally distributed data (we used the Shapiro-Wilk test to assess normality), and the $\chi^{2}$ test, Fisher's exact test, as appropriate, for categorical variables. Comparison of the different variables over the study time points between the groups and within-group comparisons between the different time points was performed using repeated measures analysis of variances for normally distributed data. Statistical significance was set at $\mathrm{P}<0.05$.

The primary outcome was peak inspiratory pressure during OLV. A power analysis suggested that a minimum sample size of 26 patients for each group would be required to achieve a significance level of $5 \%$ with a power of $80 \%$. The power was calculated from our preliminary data using an independent t-test, and the difference in mean peak inspiratory pressure between both modes of ventilation was $3 \mathrm{cmH}_{2} \mathrm{O}$, with a standard deviation of $3.8 \mathrm{cmH}_{2} \mathrm{O}$ during OLV. The dropout rate was $20 \%$, and 63 patients were sufficient. All statistical calculations were performed using the computer program SPSS version 25 (Statistical Package for the Social Science; IBM, Armonk, NY).

\section{Results}

Under the supervision of the DSMB, patient enrolment commenced on 7 August 2020. In total, 63 patients were randomly assigned to the VCV $(n=31)$ and PCV-VG $(n=32)$ groups (Fig. 1). The baseline characteristics did not differ between the groups (Table 1 ).

Peak inspiratory pressure. For the comparison of PIP between the groups, the results of repeated measures ANOVA revealed PIP at T2 and T3 were lower in the PCV-VG group than in the VCV group (T2, 20.2 \pm 2.7 $\mathrm{cmH}_{2} \mathrm{O}$ vs $\left.22.4 \pm 3.3 \mathrm{cmH}_{2} \mathrm{O} ; \mathrm{P}=0.001\right)\left(\mathrm{T} 3,23.8 \pm 3.2 \mathrm{cmH}_{2} \mathrm{O}\right.$ vs $\left.26.36 \pm 3.7 \mathrm{cmH}_{2} \mathrm{O} ; \mathrm{P}=0.01\right)$ (Fig. 2). For the comparison of PIP within groups, the Mauchly's test of sphericity, $\mathrm{P}>0.05$, indicated that the dataset satisfied 


\begin{tabular}{|c|c|c|c|}
\hline Parameters & VCV group $(n=31)$ & PCV-VG group $(n=32)$ & $P$-value \\
\hline Age (month) & $6.4[5-40.75]$ & $6.8[5.2-39.3]$ & 0.76 \\
\hline Weight (kg) & $8.3[7.5-13.25]$ & $9[7.55-12.3]$ & 0.71 \\
\hline Sex (male/female) & $25 / 6$ & $24 / 8$ & 0.59 \\
\hline \multicolumn{4}{|l|}{ Type of operation } \\
\hline Segmentectomy/wedge resection & 27 & 25 & 0.35 \\
\hline Single lobectomy & 3 & 4 & 0.72 \\
\hline Bilobectomy & 1 & 3 & 0.30 \\
\hline OLV time (min) & $87.5[71-118.75]$ & $106[60.25-125]$ & 0.44 \\
\hline Total operation time $(\mathrm{min})$ & $121.5[101.25-153.75]$ & $145[93.25-162]$ & 0.46 \\
\hline Anesthesia time (min) & 167 [144.25-192.75] & 182.0 [137.5-201.75] & 0.66 \\
\hline Respiratory rate ${ }_{\mathrm{T} 1}\left(\mathrm{~min}^{-1}\right)$ & $20.4 \pm 0.9$ & $20.1 \pm 0.4$ & 0.14 \\
\hline Respiratory rate $_{\mathrm{T} 2}\left(\mathrm{~min}^{-1}\right)$ & $26[25-27]$ & $26[25-27]$ & 0.27 \\
\hline Respiratory rate $_{\mathrm{T} 3}\left(\mathrm{~min}^{-1}\right)$ & 30 [25-30] & 25 [25-30] & 0.26 \\
\hline
\end{tabular}

Table 1. Baseline characteristics. All data are presented as mean \pm SD or median [IQR], unless otherwise specified. $V C V$ volume controlled ventilation, $P C V-V G$ pressure controlled volume guarantee ventilation, $O L V$ one lung ventilation.

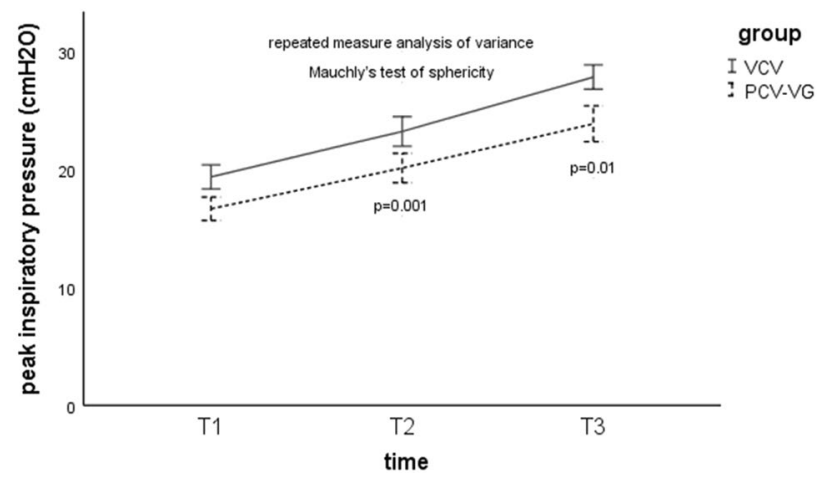

Figure 2. Peak inspiratory pressure in the two groups at different stages of the study. T1, 10 min after induction of anaesthesia in the supine position without pneumothorax; T2, 5 min after OLV commencement; T3, 5 min after complete $\mathrm{CO}_{2}$ insufflations; $V C V$ volume controlled ventilation, $P C V$ - $V G$ pressure controlled volume guaranteed ventilation. The data was presented as mean (standard deviation).

the sphericity assumption, and the results of repeated measures ANOVA revealed PIP was higher at T2 and T3 compared with $\mathrm{T} 1$ in both groups $(P=0.001)$ (Fig. 2).

Static compliance. For the comparison of static compliance between the groups, the results of repeated measures ANOVA revealed static compliance at T2 and T3 was higher in the PCV-VG group than in the VCV group (T2, $9.1 \pm 3.7 \mathrm{ml} / \mathrm{cmH}_{2} \mathrm{O}$ vs $\left.6.8 \pm 3.05 \mathrm{ml} / \mathrm{cmH}_{2} \mathrm{O} ; \mathrm{P}=0.01\right)\left(\mathrm{T} 3,7.1 \pm 3.3 \mathrm{ml} / \mathrm{cmH}_{2} \mathrm{O}\right.$ vs $4.8 \pm 2.3 \mathrm{ml} /$ $\mathrm{cmH}_{2} \mathrm{O} ; \mathrm{P}=0.01$ ) (Fig. 3).

For the comparison of static compliance within groups, the Mauchly's test of sphericity, $\mathrm{P}=0.017$, indicated that the dataset violated the sphericity assumption, therefore we accepted the result of the multivariate tests, Pillai's trace, $\mathrm{P}=0.0001$, indicating static compliance was lower at $\mathrm{T} 2$ and $\mathrm{T} 3$ compared with $\mathrm{T} 1$ in both groups $(P=0.01)$ (Fig. 3).

LUS assessment. There was no difference in LUS in the dependent lung before T0. After T0, lung aeration deteriorated, but with no difference in both groups immediately after wound closure before extubation [T4: 4 ( 2 to 6 ) vs. 4 ( 2 to 5 ) $\mathrm{Z}=-0.69, P=0.49$ ] (Fig. $4 \mathrm{a}, \mathrm{c}$ ). Lung aeration improved in both groups after surgery and returned to normal from $2.5 \mathrm{~h}$ after extubation in both groups (Fig. $4 \mathrm{~b}, \mathrm{~d}$ ). Temporal ultrasound images of the lateral chest wall of the dependent lung are shown in Fig. 4.

Postoperative pulmonary complications. Five (7.9\%) patients exhibited postoperative pulmonary complications, with no differences in the incidence between the two groups [VCV: 3 (9.7\%) vs. PCV-VG: 2 $(6.3 \%), P=0.97]$. 


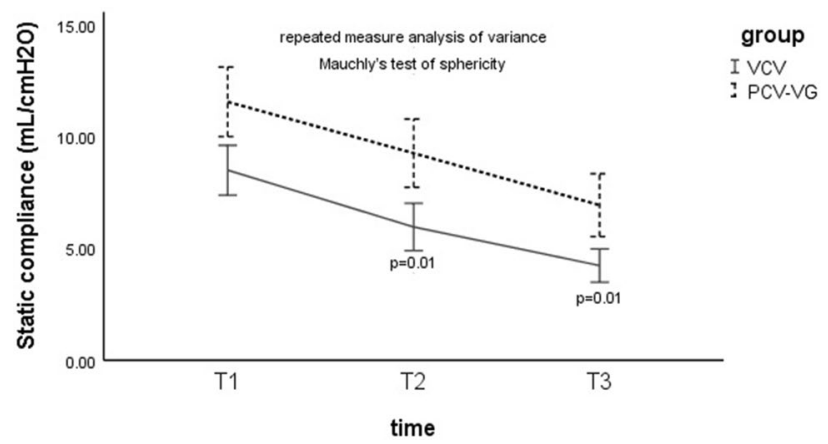

Figure 3. Static compliance in the two groups at different stages of the study. T1, 10 min after induction of anaesthesia in the supine position without pneumothorax; T2, 5 min after OLV commencement; T3, 5 min after complete $\mathrm{CO}_{2}$ insufflations; $V C V$ volume controlled ventilation, $P C V-V G$ pressure controlled volume guaranteed ventilation. The data was presented as mean (standard deviation).

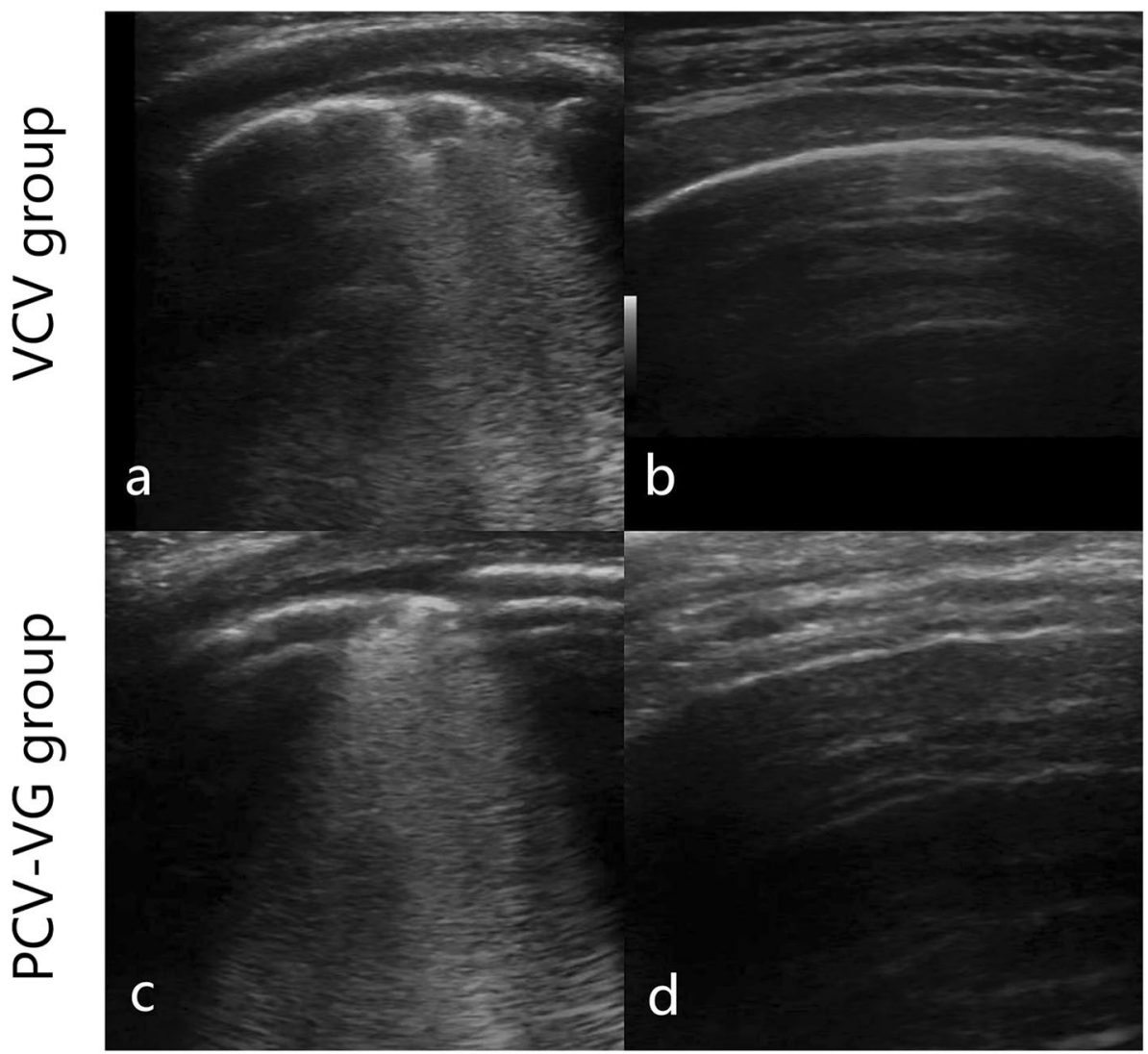

Figure 4. Lung ultrasound findings of dependent lung in the PCV-VG and VCV groups. The images were obtained from the posterior region of the dependent lung. (a) Lung aeration after surgery in VCV group. (c) Lung aeration after surgery in PCV-VG group. (b) Lung aeration $2.5 \mathrm{~h}$ after surgery in VCV group. (d) Lung aeration $2.5 \mathrm{~h}$ after surgery in PCV-VG group. $V C V$ volume controlled ventilation, $P C V$ - $V G$ pressure controlled volume guaranteed ventilation.

$\mathrm{PaO}_{2}-\mathrm{FiO}_{2}$-ratio, $\mathrm{PaCO}_{2}$, arterial $\mathrm{pH}$ and intraoperative desaturation. The data of $\mathrm{PaO}_{2}-\mathrm{FiO}_{2}-$ ratio at T2 was non-normally distributed data according to Shapiro-Wilk test. Comparison of $\mathrm{PaO}_{2}-\mathrm{FiO}_{2}-$ ratio was performed using the Mann-Whitney U-test, and there was no difference in $\mathrm{PaO}_{2}-\mathrm{FiO}_{2}$-ratio at T2 between VCV group [54 (32.2 to $62.6 \mathrm{kPa})$ ] and PCV-VG group [55.9 $(50.4$ to $63.3 \mathrm{kPa})](\mathrm{z}=-0.858 ; P=0.39)$.

The data of $\mathrm{PaO}_{2}-\mathrm{FiO}_{2}$-ratio, $\mathrm{pH}$ and $\mathrm{PaCO}_{2}$ at $\mathrm{T} 3$ were normally distributed data according to Shapiro-Wilk test. Comparisons between the groups were performed using the Student's t-test, and there was no difference in $\mathrm{PaO}_{2}-\mathrm{FiO}_{2}$-ratio at T3 between VCV group $(34.6 \pm 7.1 \mathrm{kPa})$ and $\mathrm{PCV}-\mathrm{VG}$ group $(37.9 \pm 8.0 \mathrm{kPa})(P=0.35)$. 

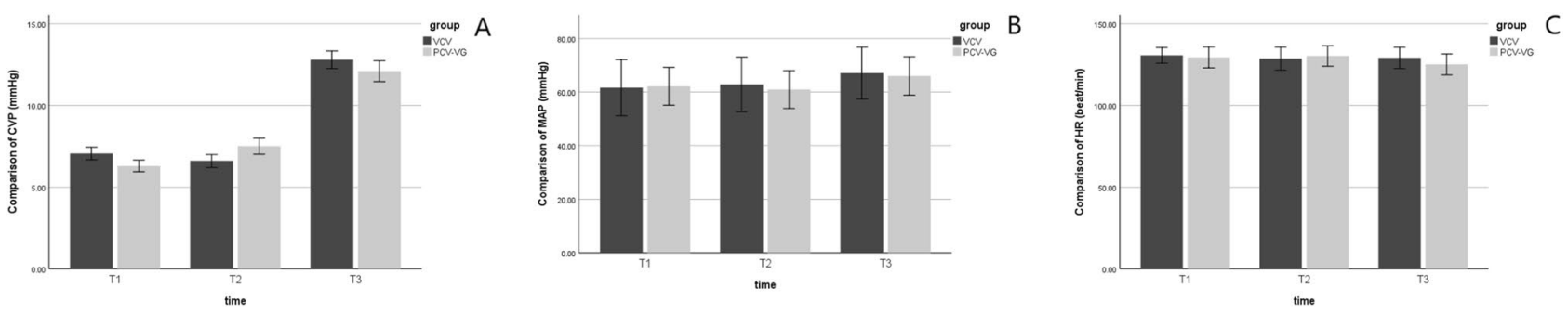

Figure 5. Comparison of haemodynamic variables at different time points. (A) CVP, central venous pressure; (B) MAP, mean arterial pressure; (C) HR, heart rate VCV, volume controlled ventilation; PCV-VG, pressure controlled volume guaranteed ventilation; T1, 10 min after induction of anaesthesia without pneumothorax; T2, 5 min after OLV commencement; T3, 5 min after complete $\mathrm{CO}_{2}$ insufflation.

However, $\mathrm{pH}$ was lower in VCV group $(7.19 \pm 0.06)$ than PCV-VG group $(7.28 \pm 0.05),(P=0.008)$. PaCO2 was higher in VCV group $(59.9 \pm 6.4 \mathrm{mmHg})$ than PCV-VG group $(51.3 \pm 7.2 \mathrm{mmHg}),(P=0.013)$.

The incidence of intraoperative desaturation was comparable in VCV group $(4 / 31,12.9 \%)$, compared with $3 / 32(9.4 \%)$ in the PCV-VG group $[\mathrm{OR}=1.43$ (0.29 to 7.0$) ; P=0.66]$.

The length of hospital stays. The length of hospital stay did not differ between the PCV-VG ( $6.5 \pm 2.1$ days) and VCV $(6.1 \pm 1.9$ days) groups. $(P=0.43)$.

Haemodynamic variables. There was no difference in the haemodynamic variables between the groups. MAP was higher at T3 in both groups compared with that at T1 and T2 $(P=0.001)$. CVP was higher at T2 and T3 than that at $\mathrm{T} 1(P=0.001)$. The heart rate was stable throughout the operation (Fig. 5).

\section{Discussion}

This randomised controlled trial revealed that PCV-VG was superior to VCV in its ability to provide ventilation with lower PIP, lower PaCO2 and higher static compliance and PH during one lung ventilation. After anaesthesia induction, lung aeration deteriorated, but with no immediate postoperative difference in both groups. Postoperative lung aeration improved and returned to normal from $2.5 \mathrm{~h}$ postextubation in both groups. No differences were observed in $\mathrm{PaO}_{2}-\mathrm{FiO}_{2}$-ratio at $\mathrm{T} 2$ and $\mathrm{T} 3$, the incidence of postoperative pulmonary complications and intraoperative desaturation and the length of hospital stay. However, its beneficial effects on different pathological situations in pediatric patients need more investigation.

Similar results have been obtained in laparoscopic ${ }^{6,7}$ and lumbar spine surgeries ${ }^{8}$. High airway pressures achieved during OLV have reportedly been associated with postpneumonectomy pulmonary oedema ${ }^{4,18}$ and acute lung injury after pneumonectomies ${ }^{19}$. Therefore, patients undergoing OLV may benefit more from lower PIP. To the best of our knowledge, evidence-based recommendations for ventilation strategies during OLV in children are lacking and this is the first randomised controlled trial to evaluate the effect of LPV combined with PCV-VG during OLV in a paediatric population.

Patients who received PCV-VG exhibited lower PIP and higher static compliance, indicating a possible association with the decreasing airflow of PCV-VG, which allows airway pressure to achieve its maximum at the beginning of inhalation and continue the entire inspiratory phase ${ }^{6}$. Continuous measurement of lung compliance and volumetric pressure automatically adjusts the air supply flow rate and air pressure ${ }^{7}$. Therefore, PCV-VG mode potentially reduces airway pressure to the greatest extent possible while ensuring ideal volume and improving lung compliance $e^{6-8,20}$.

In our study, lung aeration was comparable in both groups after surgery and was fully restored $2.5 \mathrm{~h}$ after surgery, as evaluated using LUS. This result is consistent with that of our previous study ${ }^{17}$. However, the influence of the ventilation mode can easily overlap because patient factors or other factors affect patient prognosis to a greater extent ${ }^{20}$. For instance, the lungs were manually re-expanded at the time of thoracic cavity closure. Moreover, patient may exhibit compensatory adaptations, which need to be considered ${ }^{20}$. To be more specific, patient with healthy lungs may compensate and overcome perioperative lung problems, such as lung oedema or atelectasis, but this is unlikely in patients with ALI or acute respiratory distress syndrome.

In the current study, $\mathrm{PaO}_{2}$ increased significantly in both groups at $\mathrm{T} 3$ compared to that at baseline, which might be explained by inspired oxygen concentration of 0.5 with air during TLV and 1 during OLV. Additionally, no superiority in $\mathrm{PaO}_{2}-\mathrm{FiO}_{2}$-ratio was observed regardless of the mode of ventilation, a finding that corroborates with previous studies ${ }^{6,21}$. This finding may be explained by the similarity in the mean values ${ }^{6}$. Additionally, we found no difference in the postoperative pulmonary complications (PPCs) between the two groups. This may be related to limited fluid input, plateau pressures remaining below $30 \mathrm{cmH}_{2} \mathrm{O}$ at all times ${ }^{22}$, and lung protective ventilation ${ }^{4}$. Most important, the sample was too small.

However, this study also had certain limitations. First, blinding was not conducted in investigators who were aware of the mode of ventilation. Second, we did not enroll patients with obesity or lung injuries. Therefore, its beneficial effects on different pathological situations in pediatric patients need more investigation.

In summary, $\mathrm{PCV}$-VG mode reduced the airway pressure and $\mathrm{PaCO} 2$, increased static compliance and $\mathrm{PH}$ during one lung ventilation in children undergoing pulmonary resection. 
Received: 14 September 2021; Accepted: 7 January 2022

Published online: 10 February 2022

\section{References}

1. Licker, M. J. et al. Operative mortality and respiratory complications after lung resection for cancer: Impact of chronic obstructive pulmonary disease and time trends. Ann. Thorac. Surg. 81, 1830-1837 (2006).

2. Lohser, J. Evidence-based management of one lung ventilation. Anesthesiol. Clin. 26, 241-272 (2008).

3. Fernández-Pérez, E. R. et al. Intraoperative ventilator settings and acute lung injury after elective surgery: A nested case control study. Thorax 64, 121-127 (2009).

4. Lee, J. H. et al. Lung protective ventilation during pulmonary resection in children: A prospective, single-centre, randomised controlled trial. Br. J. Anaesth. 122, 692-701 (2019).

5. Park, M. et al. Driving pressure during thoracic surgery: A randomized clinical trial. Anesthesiology 130, 385-393 (2019).

6. Assad, O. M., El Sayed, A. A. \& Khalil, M. A. Comparison of volume controlled ventilation and pressure-controlled ventilation volume guaranteed during laparoscopic surgery in Trendelenburg position. J. Clin. Anesth. 34, 55-61 (2016).

7. Lee, J. M. et al. Comparison of volume controlled, pressure-controlled, and pressure-controlled volume-guaranteed ventilation during robot-assisted laparoscopic gynecologic surgery in the Trendelenburg position. Int. J. Med. Sci. 17, 2728-2734 (2020).

8. Lee, J. M. et al. Comparison of volume controlled ventilation mode and pressure-controlled ventilation with volume-guaranteed mode in the prone position during lumbar spine surgery. BMC Anesthesiol. 19, 133 (2019).

9. Preckel, B. et al. Ten years of the Helsinki Declaration on patient safety in anaesthesiology: An expert opinion on perioperative safety aspects. Eur. J. Anaesthesiol. 37, 521-610 (2020).

10. Yan, J., Rufang, Z., Rong, W. \& Wangping, Z. Extraluminal placement of the bronchial blocker in infants undergoing capnothorax surgery: A randomized controlled study. J. Cardiothorac. Vasc. Anesth. 34, 2435-2439 (2020).

11. Templeton, T. W., Piccioni, F. \& Chatterjee, D. An update on one lung ventilation in children. Anesth. Analg. 132, 1389-1399 (2021).

12. Acosta, C. M. et al. Accuracy of transthoracic lung ultrasound for diagnosing anesthesia-induced atelectasis in children. Anesthesiology 120, 1370-1379 (2014).

13. Ghaferi, A. A., Birkmeyer, J. D. \& Dimick, J. B. Variation in hospital mortality associated with inpatient surgery. N. Engl. J. Med. 361, 136875 (2009).

14. Jammer, I. et al. Standards for defifinitions and use of outcome measures for clinical effectiveness research in perioperative medicine: European Perioperative Clinical Outcome (EPCO) definitions: A statement from the ESA-ESICM joint taskforce on perioperative outcome measures. Eur. J. Anaesthesiol. 32, 88-105 (2015).

15. Yu, X. et al. Performance of lung ultrasound in detecting perioperative atelectasis after general anesthesia. Ultrasound. Med. Biol. 42, 2775-2784 (2016).

16. Acosta, C. M. et al. Lung recruitment prevents collapse during laparoscopy in children: A randomised controlled trial. Eur. J. Anaesthesiol. 35, 573-580 (2018).

17. Zhu, C., Zhang, S., Dong, J. \& Wei, R. Effects of positive end-expiratory pressure/recruitment manoeuvres compared with zero end-expiratory pressure on atelectasis in children as tracked by ultrasound: A randomised clinical trial. Eur. J. Anaesthesiol. 38, 1026-1033 (2021).

18. Lohser, J. \& Slinger, P. Lung injury after one lung ventilation: A review of the pathophysiologic mechanisms affecting the ventilated and the collapsed lung. Anesth. Analg. 121, 302-318 (2015).

19. Yang, M. et al. Does a protective ventilation strategy reduce the risk of pulmonary complications after lung cancer surgery?: A randomized controlled trial. Chest 139, 530-537 (2011).

20. Kim, Y. S. et al. Lung ultrasound score-based perioperative assessment of pressure-controlled ventilation-volume guaranteed or volume controlled ventilation in geriatrics: A prospective randomized controlled trial. Clin. Interv. Aging. 14, 1319-1329 (2019).

21. Aldenkortt, M. et al. Ventilation strategies in obese patients undergoing surgery: A quantitative systematic review and metaanalysis. Br. J. Anaesth. 109, 493-502 (2012).

22. Amar, D. et al. Protective lung ventilation and morbidity after pulmonary resection: A propensity score-matched analysis. Anesth. Analg. 125, 190-199 (2017).

\section{Acknowledgements}

We thank our colleagues within the Department of Anesthesiology, Shanghai Children's Hospital, Shanghai, China (Yuezhen Fu, MD, PHD and Yiru Tong, MD, PHD) for technical assistance.

\section{Author contributions}

Study design/planning: R.W. and C.Z. Study conduct: R.Z. and C.Z. Data collection: S.Y. and Y.Z. Writing the paper: C.Z. and R.W. Confirming the final paper: all authors.

\section{Funding}

Funding from Special Clinical Research Project of Shanghai Municipal Health Commission (20204Y0470).

\section{Competing interests}

The authors declare no competing interests.

\section{Additional information}

Correspondence and requests for materials should be addressed to R.W.

Reprints and permissions information is available at www.nature.com/reprints.

Publisher's note Springer Nature remains neutral with regard to jurisdictional claims in published maps and institutional affiliations. 
(c) (i) Open Access This article is licensed under a Creative Commons Attribution 4.0 International cc) License, which permits use, sharing, adaptation, distribution and reproduction in any medium or format, as long as you give appropriate credit to the original author(s) and the source, provide a link to the Creative Commons licence, and indicate if changes were made. The images or other third party material in this article are included in the article's Creative Commons licence, unless indicated otherwise in a credit line to the material. If material is not included in the article's Creative Commons licence and your intended use is not permitted by statutory regulation or exceeds the permitted use, you will need to obtain permission directly from the copyright holder. To view a copy of this licence, visit http://creativecommons.org/licenses/by/4.0/.

(C) The Author(s) 2022 